\title{
Instrumentación de muletas de antebrazo para uso en la monitorización de marcha y movimiento
}

\author{
Marien Narváez \\ marien.cristina.narvaez@upc.edu \\ Joan Aranda \\ joan.aranda@upc.edu
}

\begin{abstract}
Resumen
Los dispositivos sensorizados en el ámbito de la rehabilitación física, proveen información cuantificable que le permiten al terapeuta conocer de forma objetiva el estado o evolución de la terapia del paciente. Para este estudio, fueron instrumentadas dos muletas de antebrazo que permitieron monitorear tres tipos de marcha de cinco sujetos sanos. Cada prototipo es capaz de medir la fuerza axial aplicada por las extremidades superiores a la muleta y la orientación y aceleración de la misma, para cada ciclo de marcha en un patrón preestablecido. Con los datos obtenidos se analizaron características críticas de la marcha que permitirían potencialmente cuantificar el desempeño de la marcha y determinar la evolución del paciente en un proceso de rehabilitación. Este sistema se presenta como una alternativa para el análisis de marcha con muletas y la individualización de la terapia, a través de la monitorización de los pacientes en su proceso de rehabilitación.
\end{abstract}

Palabras clave: Muletas instrumentadas, tecnologías de asistencia, monitorización de la marcha, análisis de marcha

\section{INTRODUCCIÓN}

En los últimos años, la monitorización de las actividades de rehabilitación se ha incrementado notablemente debido a la evolución de los sistemas electrónicos y de comunicación en paralelo a las necesidades en la terapia física [1]. En este contexto, la evaluación confiable y funcional de la marcha humana y sus alteraciones implica el desarrollo de diversos mecanismos o instrumentos, tales como plataformas para medición de fuerzas, sistemas para análisis de movimiento 3D o sistemas de captura de datos usando sensores localizados sobre la persona, entre otros $[2,3]$.

Los dispositivos sensorizados proveen datos objetivos que pueden permitir al paciente y al terapeuta cuantificar la progresión en la terapia, además de ayudar en la toma de decisiones precisas para adaptar el tratamiento de acuerdo con los paráme- tros medidos y estandarizar el protocolo del tratamiento que permita obtener resultados confiables, comparables y eficaces [2].

En el ámbito de la terapia física, las muletas de antebrazo son dispositivos diseñados para asistir en la marcha de personas cuando una de las extremidades inferiores requiere de asistencia y se utilizan individualmente o por pares [4]. Es bien sabido que el uso incorrecto de las muletas puede extender el proceso de rehabilitación y causar dolor o futuras lesiones $[5,6]$.

Estudios previos $[8,7,9]$, muestran diversos prototipos para monitorizar actividades de rehabilitación a través de muletas instrumentadas. Estos, generalmente han sido diseñados para medir las fuerzas ejercidas por el paciente y la orientación y posición de las muletas. Sin embargo estos estudios no comparan los cambios en la marcha en la ejecución de diferentes patrones. El objetivo de este estudio es monitorizar la marcha en muletas y analizar los cambios en diferentes parámetros espacio- temporales para 3 patrones de marcha diferentes. En la primera parte de este artículo se describe la implementación del sistema, compuesto por sensores de bajo costo e instalado sobre un par de muletas de antebrazos, para medir la fuerza normal aplicada por las extremidades superiores y ángulos relevantes en el movimiento de la muleta. La siguiente sección trata de la marcha con muletas y los parámetros relevantes en este contexto. Más adelante se presentan los experimentos realizados para medir las variables de interés. Por último se discuten los resultados obtenidos y se plantean las conclusiones.

\section{MULETAS INSTRUMENTADAS}

Para este trabajo fueron instrumentadas un par de muletas con el objetivo de medir la fuerza axial, ángulos de orientación (inclinación y giro) de la muleta y la aceleración de la misma. Los ángulos son relativos a la muleta y el suelo. Estas variables son transferidas en tiempo real a un ordenador vía Bluetooth. La Figura 1 muestra un esquema del sistema propuesto. 


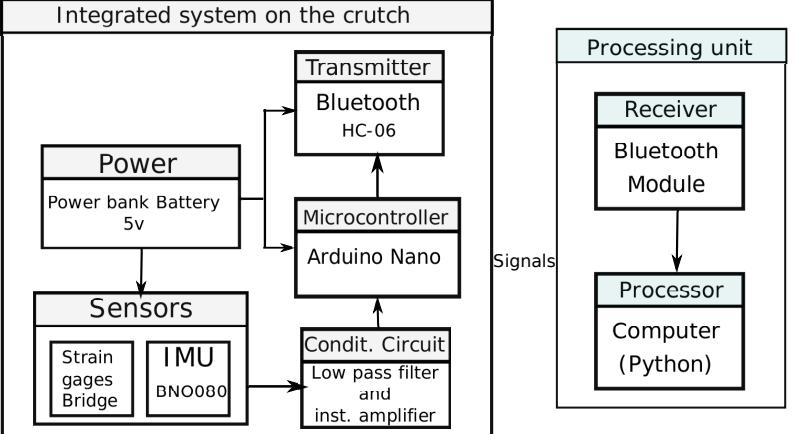

Figura 1: Sistema propuesto para cada muleta

\subsection{IMPLEMENTACIÓN DEL SISTEMA}

La fuerza axial fue medida usando galgas extensiométricas instaladas en el mango de la muleta en una configuración de puente Wheatston. Estos sensores fueron calibrados usando cargas conocidas para obtener valores de fuerza en Newtons, mediante ensayos de pesos muertos en el mango de la muleta. Por otra parte, la orientación y aceleración de la muleta fueron medidas a través de una Unidad de Medición Inercial (IMU) BNO080 (Sparkfun). Las IMUs fueron calibradas a partir de los ejes establecidos (ejes $z$ y $x$ ) como se muestra en la imagen 2.

\subsubsection{Adquisición de datos}

Estas señales son adquiridas, filtradas y amplificadas usando un circuito de acondicionamiento que consiste en un filtro pasabajos y un amplificador de instrumentación (INA 126). Luego, a través de un microcontrolador estas señales son acondicionadas para ser transmitidas vía Bluetooth.

Los componentes fueron ensamblados en un circuito impreso alimentado por una batería externa de $5 \mathrm{~V}, 2600 \mathrm{mAh}$ para cada muleta. El circuito está ubicado en una caja instalada debajo del mando de la muleta, como puede verse en la imagen 2. La información es procesada y almacenada en tiempo real en un ordenador estándar con un receptor Bluetooth externo. Los datos son adquiridos con una frecuencia de muestreo de $75 \mathrm{~Hz}$. El proceso para la instrumentación de cada muleta fue el mismo.

\subsection{PROCESAMIENTO DE LOS DATOS}

El procesamiento de los datos obtenidos consiste en tres etapas: filtrado, segmentación de las fases de la marcha y obtención de las variables de interés.

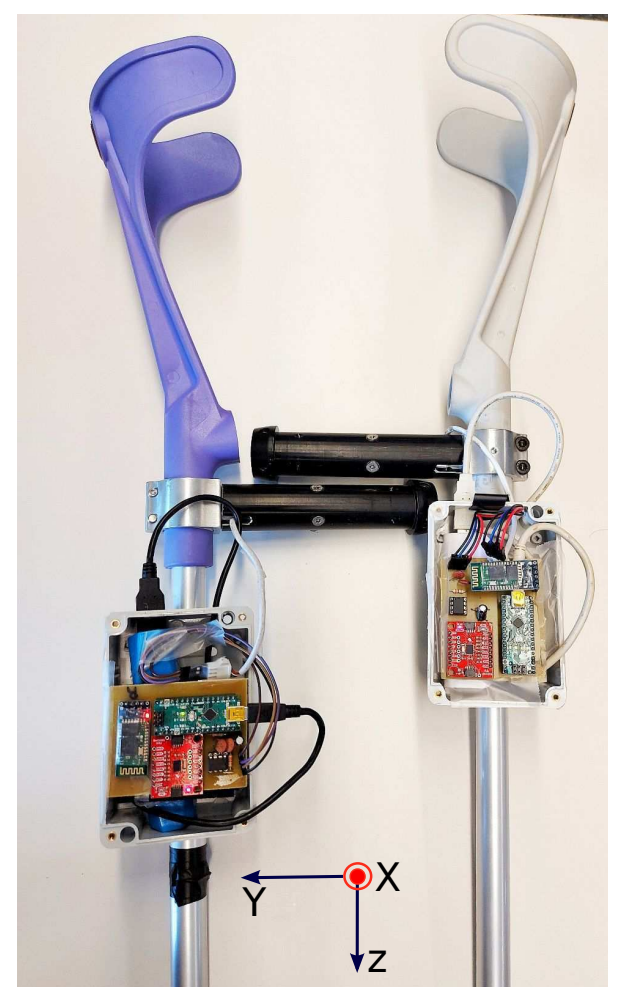

Figura 2: Muletas instrumentadas

Para atenuar las aceleraciones dinámicas medidas por las IMUs, fue implementado un filtro de media móvil, mientras que la señal de fuerza fue suavizada a través de un filtro Gausiano para reducir los efectos del ruido analógico.

Posteriormente y tomando en cuenta la literatura estudiada, la señal es segmentada en las fases balanceo (Swing) y apoyo (Stance) de la marcha. Para ello, usamos como referencia el signo de la derivada del ángulo de inclinación en el sentido de la marcha (respecto al eje $\mathrm{x}$ ). El proceso de segmentación de la señal para cada tipo de marcha se describe detalladamente en [10].

Cada ejercicio de marcha fue segmentado en ventanas de 5 ciclos, donde se hallaron valores medios para cada variable de interés (fuerza máxima, inclinación y tiempos por ciclo de marcha). A partir de estas variables se calcularon parámetros característicos que son necesarios para el análisis de marcha.

\section{ANÁLISIS DE MARCHA CON MULETAS}

Tomando en cuenta estudios previos, el análisis de la marcha con muletas incluye la medición del rendimiento del usuario (respuestas físicas y fisiológicas) así como aspectos relacionados con la cinemática de la locomoción [16]. Por consiguiente es significativo el estudio de las fuerzas aplicadas 
por las extremidades superiores, las cuales son responsables del desplazamiento del cuerpo en cada ciclo de marcha. También el análisis de variables como, ángulos de posición de la muleta, ratios de fuerza y tiempo entre las fases de swing/stance , velocidad y aceleración, entre otros. La fuerza ejercida, los ángulos de las muletas y la relación entre las fases dentro del ciclo de la marcha son parámetros esenciales para examinar el comportamiento de la marcha con muletas en función del patrón de marcha. Medidas de tiempo, tales como el tiempo de contacto pueden indicar un cambio en el patrón de marcha. Los ángulos de las articulaciones muestran la trayectoria de la muleta durante la marcha y la relación de fase y la relación swing/ stance compara el porcentaje relativo de cada postura o porción de swing durante un paso [16]. La marcha humana incluye progresiones cíclicas y repetitivas para cada extremidad inferior, alternadas entre la fase de balanceo y apoyo. Sin embargo factores como cambios fisiológicos, enfermedades neurodegenerativas, lesiones, etc. pueden comprometer la función de la marcha y afectar la capacidad del sistema neuromuscular para mantener un nivel constante de movilidad. Por lo tanto, evaluar las desviaciones de la marcha es una medida útil para la salud en general, la calidad de vida y como predictor de deterioro físico y cognitivo, entre otras [14].

Una de las formas de medir esta desviación es por medio del parámetro asimetría. Esta característica está asociada con la cantidad de divergencia entre el lado derecho e izquierdo del cuerpo. Asimetrías en la marcha pueden reflejar diferencias funcionales en la contribución de cada miembro en la propulsión y el control durante la locomoción.

Existen diferentes métodos $[14,15,13]$ para determinar la asimetría en la marcha y que incluyen diversos parámetros. Para este trabajo fue escogido el Índice de Simetría o Índice de Robinson 1, el cual ha sido usado en trabajos como [15], para cuantificar la asimetría de las fuerzas de reacción.

$$
S I=2 \frac{\left|X_{L}-X_{R}\right|}{X_{L}+X_{R}}
$$

En la ecuación 1,

$$
X_{L}, X_{R},>=0
$$

corresponde a la variable de la cual se pretende establecer el índice, L para el lado izquierdo y $\mathrm{R}$ para el lado derecho. Este índice fue utilizado en este trabajo para comparar las fuerzas máximas aplicadas por cada extremidad y los grados de inclinación máximos alcanzados por la muleta.

\subsection{Tipos de marcha}

Existen diferentes formas de caminar con muletas, cuya prescripción depende de la lesión o la condición física de un paciente. Los patrones de marcha dependen de la capacidad del usuario para mover los pies recíprocamente, tolerar cargas en cada pierna, levantar el cuerpo del suelo extendiendo los codos y presionando las manos y simultáneamente mantener el equilibrio [16]. En $[10,16]$ son descritos los tipos de marcha con muletas más utilizados. En este trabajo consideramos los siguientes tipos de marcha:

- Dos puntos $(2 \mathrm{p})$ : Se levantan simultáneamente una muleta y el pie opuesto, y luego la otra muleta y el pie contrario (pacientes con debilidad en ambas piernas, pero fuerza en ambos brazos).

- Tres puntos (3p): Se adelantan primero las dos muletas, seguido de la pierna en la que se tenga menos fuerza, y por último, el otro pie (pacientes que tienen toda la fuerza en una sola pierna).

- Cuatro puntos (4p): Se adelanta una de las muletas, luego el pie opuesto, seguido de la otra muleta, y finalizando con el otro pie (pacientes que tienen poca fuerza en las dos piernas).

En la Figura 3 se presenta la secuencia de los 3 patrones de marcha a ser analizados.

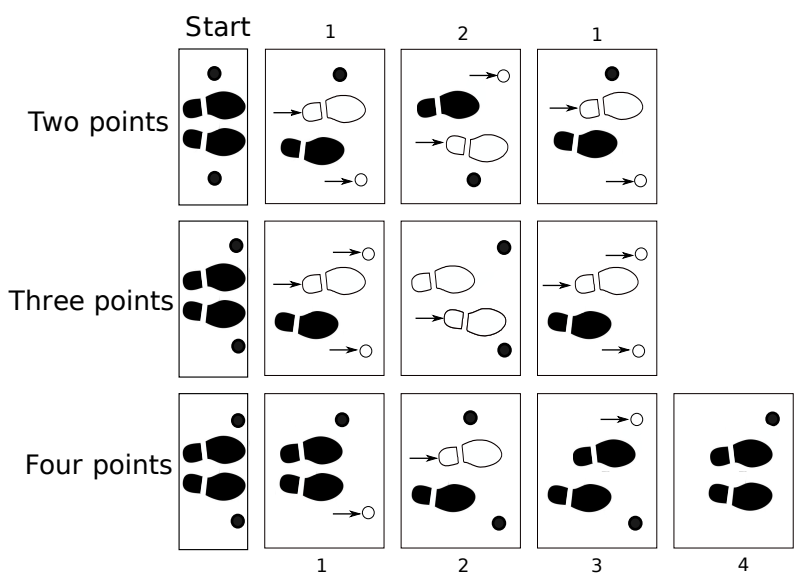

Figura 3: Secuencia de pasos para 3 patrones de marcha. Las áreas sombreadas representan la extremidad apoyada y sobre la cual recae el peso. Los números determinan el período en la secuencia de la marcha, cuando los puntos de contacto tocan el piso síncronamente. Las flechas indican el avance del pie o de la muleta. Imagen modificada de $[16]$ 


\section{PROTOCOLO EXPERIMENTAL}

Para la validación del sistema implementado en ambas muletas y la monitorización de diferentes tipos de marcha fueron realizados una serie de experimentos. En este estudio participaron 5 sujetos (edad promedio $28.2 \pm 3.56$ años, estatura $170 \pm$ $6.78 \mathrm{~cm}, 66.8 \pm 9.65 \mathrm{~kg}$ ) sin condiciones ortopédicas o dolor que modificaran su marcha. Ninguno de ellos había usado muletas previamente, por lo que fueron instruidos para ejecutar correctamente las marchas de 2, 3 y 4 puntos siguiendo la guía [12] y videos explicados por fisioterapeutas. Esto, para ser ejecutado en una distancia de 10 metros, lo que habitualmente genera un mínimo de 15 ciclos por ejercicio.

Los participantes caminaron 3 veces en cada uno de los patrones de marcha, simulando una lesión en la pierna derecha, por lo que no podían apoyar todo su peso en la misma. Entre cada ejercicio tenían un tiempo de descanso de 3 minutos.

\section{RESULTADOS Y DISCUSIÓN}

Los resultados presentados a continuación corresponden a 5 sujetos, quienes presentan diferencias antropométricas significativas. Los sujetos 1,3 y 5 son mujeres con peso de $62.5 \mathrm{~kg} 55 \mathrm{~kg}$ y 64.2 $\mathrm{kg}$, con estaturas de $162 \mathrm{~cm}$ y $166 \mathrm{~cm} \mathrm{y} 163 \mathrm{~cm}$ respectivamente. Los sujetos 2 y 4 son hombres de $80 \mathrm{~kg}$ y $68 \mathrm{~kg}$ con estatura de $180 \mathrm{~cm} \mathrm{y} 170 \mathrm{~cm}$ respectivamente.
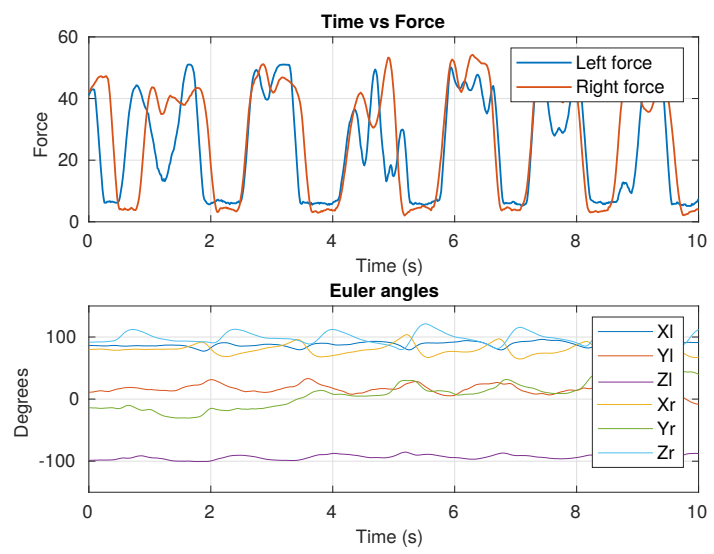

Figura 4: Medidas de fuerza y ángulos para 10s de marcha del Sujeto 1 ejecutando la marcha de 3 puntos. La gráfica superior representa la fuerza normal ejecutada sobre cada muleta. La gráfica muestra el movimiento de las muletas medido en grados, respecto a los ejes X, Y y Z. Las letras 1 y r, corresponden a izquiera y derecha respectivamente.
La gŕafica 4 muestra las señales capturadas para 10 s de marcha con ambas muletas, ejecutando la marcha de 3 puntos. En este patrón de marcha ambas muletas avanzan en simultáneo lo cual puede notarse en los ciclos presentados en la imagen superior, los cuales corresponden aproximadamente a los mismos tiempos. Así mismo puede verse los ángulos de inclinación de la muleta medidos respecto al eje X, y dada la contra lateralidad de las muletas, en cada ciclo pueden verse movimientos opuestos. Con estos resultados, para este estudio se omitieron los ángulos medidos respecto a $\mathrm{Z}$ y Y por no representar medidas significativas. La Tabla 1, presenta los datos promedio para 3 tipos de marcha ejecutados por 5 individuos. De acuerdo con las medidas realizadas por el sistema en las muletas, la literatura estudiada y la asesoría por parte del personal médico, fueron considerados relevantes los parámetros: Fuerza Máxima, Inclinación máxima, y los ratios de fuerza y tiempo en las fases de swing/stance

Para los 3 individuos pueden determinarse diferencias entre las fuerzas y las inclinaciones máximas para el lado derecho e izquierdo.

Según la Tabla 1 en general, cuando el ángulo máximo de inclinación aumenta, el ratio swing/stance también lo hace, esto quiere decir que la persona emplea más tiempo en la fase de balanceo (swing). De acuerdo con estudios previos [16], sujetos en alguna condición de discapacidad física ejecutan la fase de apoyo (stance) en mayor tiempo debido a la falta de activación de la cadera y por tanto una flexión más lenta de la misma. También puede notarse que el swing/stance en general es mayor en la marcha de 4 puntos. Durante los experimentos pudo verse que este patrón es el que tiene más dificultad en su ejecución y el que se realiza de forma más lenta, pues requiere de coordinación de extremidades inferiores y superiores en cada paso. Dado que la marcha de 3 puntos implica el movimiento simultáneo de ambas muletas y el cuerpo se inclina hacia adelante más que con los otros patrones, las mayores fuerzas para los sujetos de estudio fueron aplicadas en este patrón de marcha.

La Tabla 1, permite establecer cuáles son los patrones de marcha con mayores asimetrías para cada individuo. Esta variable es cuantificada en la Tabla 2, que presenta los porcentajes de asimetría entre la fuerza y la inclinación máxima para cada caso. En estos primeros cálculos de asimetría fueron escogidas para análisis las características fuerza máxima e inclinación máxima. La fuerza máxima es un paramétro crítico para evaluar la ejecución de la marcha con las muletas, ya que permite ver la distribución del peso aplicado por 
Cuadro 1: Comparación de parámetros de marcha para 3 sujetos. Las letras L y R para cada tipo de marcha corresponden a izquierda y derecha respectivamente. Los valores presentados son valores promedio.

\begin{tabular}{c|c|c|c|c|c|c|c}
\hline Sujeto & Parámetro & 2p L & 2p R & 3p L & 3p R & 4p L & 4p R \\
\hline \multirow{5}{*}{ S1 } & Fuerza máx (N) & 46.73 & 47.98 & 54.07 & 53.01 & 51.39 & 54.38 \\
& Inclinación (o) & 24.08 & 26.08 & 32.01 & 24.66 & 27.23 & 25.14 \\
& Ratio swing/stance (T) & 0.46 & 0.66 & 0.59 & 0.62 & 0.69 & 0.69 \\
& Ratio swing/stance (F) & 0.2 & 0.18 & 0.24 & 0.32 & 0.7 & 0.69 \\
\hline \multirow{5}{*}{ S2 } & Fuerza máx (N) & 80.68 & 102.25 & 100.07 & 96.03 & 83.62 & 78.69 \\
& Inclinación (o) & 14.94 & 12.11 & 17.02 & 14.9 & 17.26 & 18.68 \\
& Ratio swing/stance (T) & 0.42 & 0.46 & 0.49 & 0.41 & 0.74 & 0.67 \\
& Ratio swing/stance (F) & 0.19 & 0.2 & 0.47 & 0.29 & 0.41 & 0.46 \\
\hline \multirow{5}{*}{ S3 } & Fuerza máx (N) & 48.67 & 44.67 & 59.6 & 60.47 & 49.37 & 50.53 \\
& Inclinación (o) & 15.21 & 17.79 & 17.61 & 17.59 & 17.25 & 20.35 \\
& Ratio swing/stance (T) & 0.37 & 0.66 & 0.7 & 0.75 & 0.63 & 0.62 \\
& Ratio swing/stance (F) & 0.19 & 0.23 & 0.38 & 0.43 & 0.49 & 0.38 \\
\hline \multirow{5}{*}{ S4 } & Fuerza máx (N) & 55.88 & 51.5 & 60.46 & 59.54 & 59.88 & 55.33 \\
& Inclinación (o) & 22.27 & 20.55 & 18.44 & 19.58 & 22.42 & 24.84 \\
& Ratio swing/stance (T) & 0.47 & 0.49 & 0.58 & 0.63 & 0.66 & 0.71 \\
& Ratio swing/stance (T) & 0.22 & 0.24 & 0.35 & 0.29 & 0.68 & 0.65 \\
\hline \multirow{5}{*}{ S5 } & Fuerza máx (N) & 47.31 & 49.94 & 60.03 & 65.81 & 60.28 & 63.29 \\
& Inclinación (o$)$ & 17.27 & 16.12 & 21.99 & 17.26 & 17.25 & 25.42 \\
& Ratio swing/stance (T) & 0.52 & 0.68 & 0.62 & 0.62 & 0.63 & 0.65 \\
& Ratio swing/stance (F) & 0.24 & 0.28 & 0.39 & 0.33 & 0.64 & 0.66 \\
\hline
\end{tabular}

Cuadro 2: Valores de asimetría expresados en porcentajes usando el índice de Robison 1

\begin{tabular}{ccccc}
\hline Sujeto & Parámetro & $\mathbf{2 p}$ & $\mathbf{3 p}$ & $\mathbf{4 p}$ \\
\hline \multirow{2}{*}{ S1 } & Fuerza & 2.63 & 1.97 & 5.65 \\
& Inclinación & 7.97 & 25.95 & 7.98 \\
\hline \multirow{2}{*}{ S2 } & Fuerza & 23.58 & 4.12 & 6.07 \\
& Inclinación & 20.91 & 13.28 & 7.90 \\
\hline \multirow{2}{*}{ S3 } & Fuerza & 8.57 & 1.45 & 2.32 \\
& Inclinación & 15.64 & 0.06 & 16.47 \\
\hline \multirow{2}{*}{ S4 } & Fuerza & 8.16 & 1.53 & 7.90 \\
& Inclinación & 8.03 & 0.06 & 10.24 \\
\hline \multirow{2}{*}{ S5 } & Fuerza & 5.41 & 9.19 & 4.87 \\
& Inclinación & 6.89 & 24.1 & 32.57 \\
\hline
\end{tabular}

las extremidades superiores y de esta forma determinar cambios en la terapia y evitar lesiones, entre otros aspectos que corresponden al análisis clínico. La inclinación permite establecer el inicio y fin de un ciclo de marcha, de acuerdo con el análisis hecho en trabajos previos.

De acuerdo con la tabla 2 los valores de asimetría varían de modo importante para cada sujeto, en un tipo de marcha. Por ejemplo el Sujeto 2, quien presenta características de peso y altura mayores comparado con los otros participantes, tuvo difi- cultad en la ejecución del patrón de dos puntos, tanto en la distribución de la fuerza como en el movimiento de la muleta. En general, el parámetro con mayores valores de asimetría es el grado de inclinación máximo de la muleta ejecutado por cada sujeto, relacionado con el movimiento en la ejecución del patrón de marcha y con la postura que podría adoptar la persona con las muletas. Por otra parte, al parámetro de la fuerza máxima le corresponden los valores más bajos de simetría, con lo que puede determinarse que los sujetos distribuyeron su fuerza en ambas extremidades de forma más efectiva. Al tratarse de individuos sin lesiones reales puede deducirse que la distribución de fuerza incluyó las extremedidades inferiores y no se recibió la mayor parte del apoyo en los brazos. Ya que de acuerdo con la literatura y estudios pasados, la fuerza aplicada en el mango de las muletas corresponde a valores entre $111 \%$ y $120 \%$ del peso corporal aproximadamente.

\subsection{CONCLUSIONES Y TRABAJOS FUTUROS}

Entre las principales motivaciones de este trabajo, se encuentra la inidvidualización de la terapia en el proceso de rehabilitación de usuarios con muletas. Este trabajo presentó la instrumentación de 
un par de muletas para uso en la monitorización de la marcha y movimiento en sujetos sanos. De acuerdo con las asimetrías medidas más la observación directa de los ejercicios, los profesionales clínicos podrían determinar el nivel de la terapia en la que se encuentra el paciente, sugerir cambios en la ejecución del ejercicio o cambiar el patrón de marcha de acuerdo con la condición tratada. A pesar de que las pruebas hasta ahora han sido en el ambiente de laboratorio, los resultados obtenidos son útiles para comenzar experimentos dentro del ambiente clínico, en el que pueda monitorizarse los ejercicios bajo supervisión del terapeuta.

Los individuos en este estudio simularon una lesión para ejecutar la marcha con muletas, y aunque fueron observados y entrenados, presentaron asimetrías de diferentes proporciones en la ejecución. Sin embargo, estos dos parámetros no son suficientes para evaluar la condición del paciente, puesto que los fallos en el desempeño en los patrones de marcha pueden deberse a diferentes factores. Por tanto se hace necesario la inclusión de más variables características de la marcha. En nuestras próximas investigaciones, usaremos el sistema en personas en condición de discapacidad física real, donde podrá hacerse una evaluación de la marcha, que permita ver la ejecución de los patrones de marcha y a largo plazo ver la evolución de los pacientes. Los trabajos futuros incluirán además la correlación de índices de simetría con condiciones y lesiones particulares, junto con el análisis de otras características en la marcha, tales como velocidad y aceleración.

\section{Agradecimientos}

Agradecemos a los voluntarios por la participación en los experimentos y al aŕea de investigación del Hospital Sant Joan de Deu por la asesoría.

\section{English summary}

\section{INSTRUMENTED FOREARM CRUTCHES FOR USING IN GAIT AND MOVEMENT MONITORING}

\author{
Abstract \\ Sensorized devices in physical rehabilita- \\ tion provide quantifiable information that \\ allows the therapist to objectively know the \\ status or evolution of the patient's therapy. \\ For this study, two forearm crutches were \\ instrumented that allowed monitoring three \\ types of gait of five healthy subjects. Each \\ prototype can measure the axial force ap- \\ plied by the upper extremities to the crutch
}

and its orientation and position for each gait cycle within a pre-established pattern. The results obtained show critical characteristics to analyse and evaluate the gait evolution and determine possible errors in the user's performance. This system is an alternative for gait analysis using crutches and a tool for individualising therapy through the monitoring of patients in their rehabilitation process.

Keywords: Instrumented crutches, Assistive technologies, Gait monitoring, Gait analysis

\section{Referencias}

[1] Do Nascimento L. M. S., Bonfati L. V., Freitas M. L. B., Mendes Junior J. J. A., Siqueira H. V., and Stevan S. L., "Sensors and systems for physical rehabilitation and health monitoring - a review," Sensors (Switzerland), vol. 20, no. 15, pp. 1-28, 2020.

[2] Muro-de-la-Herran A., García-Zapirain B., and Méndez-Zorrilla A., "Gait analysis methods: An overview of wearable and nonwearable systems, highlighting clinical applications," Sensors (Switzerland), vol. 14, no. 2, pp. 3362-3394, 2014.

[3] Chamorro-Moriana G., Ridao-Fernández C. , Ojeda J. , Benítez-Lugo M., and Sevillano J. L., "Reliability and validity study of the Chamorro Assisted Gait Scale for people with sprained ankles, walking with forearm crutches," PLoS One, vol. 11, no. 5, pp. 1-12, 2016.

[4] Edelstein, J.: 36 - canes, crutches, and walkers.Atlas of Orthoses and Assistive Devices, 5th Edn. Elsevier Inc. (2019)

[5] Sardini, E., Serpelloni, M., Lancini, M.: Wireless instrumented crutches for force and movement measurements for gait monitoring. IEEE Trans. Instrum. Meas. 64(12), 3369-3379 (2015)

[6] Capecci, D., Kim, S.H., Reed, K.B., Handzic, I.: Crutch tip for swing-through crutch walking control based on a kinetic shape. IEEE International Conference on Rehabilitation Robotics (ICORR), pp. 612-617 (2015) 
[7] Merrett G. V. , Ettabib M. A. , Hallett C. Peters, G., White N. M., "Augmenting forearm crutches with wireless sensors for lower limb rehabilitation," Measurement Science and Technology, vol. 21, no. 12, 2010.

[8] G. Chamorro-Moriana, J. L. Sevillano, and C. Ridao-Fernández, "A compact forearm crutch based on force sensors for aided gait: Reliability and validity," Sensors (Switzerland), vol. 16, no. 6, pp. 1-15, 2016.

[9] I. Sesar, A. Zubizarreta, I. Cabanes, E. Portillo, J. Torres-Unda, and A. Rodriguez- Larrad, "Instrumented Crutch Tip for Monitoring Force and Crutch Pitch Angle," Sensors, vol. 19, no. 13, p. 2944, 2019.

[10] Narváez M. and Aranda J. , "Gait Patterns Monitoring Using Instrumented Forearm Crutches," Computers Helping People with Special Needs, 2020, pp. 402-410.

[11] F. Rasouli and K. B. Reed, "Walking assistance using crutches: A state of the art review," J. Biomech., vol. 98, Jan. 2020.

[12] Oxford University Hospitals NHS Trust. (2015). Using Elbow Crutches Instruction for patients.

[13] Błazkiewicz, M., Wiszomirska, I., Wit, A. (2014). Comparison of four methods of calculating the symmetry of spatial-temporal parameters of gait. Acta of Bioengineering and Biomechanics, 16(1), 29-35.

[14] Viteckova, S., Kutilek, P., Svoboda, Z., Krupicka, R., Kauler, J., Szabo, Z. (2018). Gait symmetry measures: A review of current and prospective methods. Biomedical Signal Processing and Control, 42, 89-100.

[15] Alves, S. A., Ehrig, R. M., Raffalt, P. C., Bender, A., Duda, G. N., Agres, A. N. (2020). Quantifying Asymmetry in Gait: The Weighted Universal Symmetry Index to Evaluate 3D Ground Reaction Forces. Frontiers in Bioengineering and Biotechnology, 8(October).

[16] F. Rasouli and K. B. Reed, "Walking assistance using crutches: A state of the art review," J. Biomech., vol. 98, Jan. 2020.

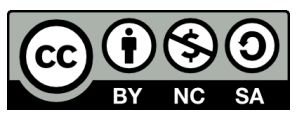

(C) 2021 by the authors. Submitted for possible open access publication under the terms and conditions of the Creative Commons Attribution CC BY-NC-SA 4.0 license (https://creativecommons.org/licenses/by-ncsa/4.0/deed.es). 